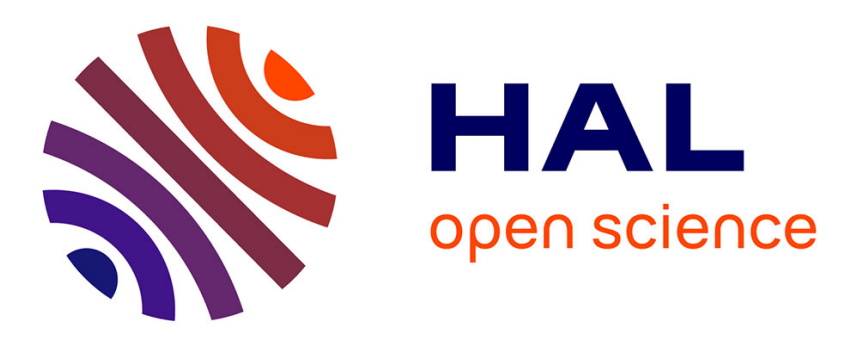

\title{
Smart microrobots for mechanical cell characterization and cell convoying.
}

\author{
Medhi Boukallel, Michaël Gauthier, Michaël Dauge, Emmanuel Piat, Joël
}

Abadie

\section{> To cite this version:}

Medhi Boukallel, Michaël Gauthier, Michaël Dauge, Emmanuel Piat, Joël Abadie. Smart microrobots for mechanical cell characterization and cell convoying.. IEEE Transactions on Biomedical Engineering, 2007, 54 (8), pp.1536-1540. hal-00179481

\section{HAL Id: hal-00179481 \\ https://hal.science/hal-00179481}

Submitted on 15 Oct 2007

HAL is a multi-disciplinary open access archive for the deposit and dissemination of scientific research documents, whether they are published or not. The documents may come from teaching and research institutions in France or abroad, or from public or private research centers.
L'archive ouverte pluridisciplinaire HAL, est destinée au dépôt et à la diffusion de documents scientifiques de niveau recherche, publiés ou non, émanant des établissements d'enseignement et de recherche français ou étrangers, des laboratoires publics ou privés. 


\title{
Smart Microrobots for Mechanical Cell Characterization and Cell Convoying
}

\author{
M. Boukallel ${ }^{\ddagger}$, M. Gauthier ${ }^{\star}$, M.Dauge ${ }^{\star}$, E. Piat ${ }^{\star}$, J. Abadie $^{\star}$. \\ $\ddagger$ Robotics Laboratory of Paris (LRP - FRE 2507) \\ 18, route du Panorama - 92265 Fontenay aux Roses - France \\ Tel: +33 (0) 146548935 - Fax: +33 (0) 146547299 \\ Website: http://lrp6.robot.jussieu.fr/lrp6/eng/ \\ E-mail: boukallel@ robot.jussieu.fr \\ * Laboratory of Automation of Besançon (LAB - UMR CNRS) \\ 24, rue Alain Savary - 25000 Besançon - France \\ Tel: +33 (0) 381402810 - Fax: +33 (0) 381402809 \\ Website: http://www.lab.cnrs.fr \\ E-mail: gauthier@ens2m.fr
}

\begin{abstract}
This paper deals with the effective design of smart microrobots for both mechanical cell characterization and cell convoying for in vitro fertilization. The first microrobotic device was developed to evaluate oocyte mechanical behavior in order to sort oocytes. A multi-axial micro-force sensor based on a frictionless magnetic bearing was developed. The second microrobotic device presented is a cell convoying device consisting of a wireless micropusher based on magnetic actuation. As wireless capabilities are supported by this microrobotic system, no power supply connections to the micropusher are needed. Preliminary experiments have been performed regarding both cell transporting and biomechanical characterization capabilities under in vitro conditions on human oocytes so as to demonstrate the viability and effectiveness of the proposed setups.
\end{abstract}

Index Terms-Microrobotics, in vitro fertilization process, mechanical cell characterization, cell convoying.

ORTING fertilized oocytes (embryos) in order to select a good pattern for the transfer process is an important issue. The quality of the transferred embryos and the quality of the oocytes is a crucial parameter of the resulting embryo quality. To date, fertilized oocytes are sorted based on the visual optical microscope information relayed to the operator for evaluation. Experimental observations have shown that cell morphological transformation is observed from 48 to 72 hours after the fertilization process. Thus, fertilization failures are usually detected only at an advanced stage when the fertilized oocytes fail to divide or stop their development. Some recent studies [1][2] have shown that strong mechanical behavior modifications are observed a few hours after the fertilization process. Using a new criterion for oocyte sorting associated with visual information should improve oocyte sorting for earlier failure detection procedures.

Repeated micromanipulation tasks required in the in vitro fertilization process are commonly carried out by highly skilled operators. Since these repeated manipulation are important, low efficiency tasks may be achieved even by experienced human operators. Oocytes are usually manipulated during the in vitro fertilization process using a micro-pipette based on suction method. Human oocytes are fragile and must be manipulated carefully in order to reduce damage to their external or internal membranes. The development of an automated and supervised system reducing involvement of human operators based on a minimally invasive approach during the crucial steps of the fertilization process is a suitable solution.

In the recent years, the robotics and microrobotics fields have played an important role in the development of a dedicated systems for microbiology applications. Many efforts have been devoted to development towards a high efficiency artificial fertilization process [3-11]. Despite these research efforts, studies focus mainly on a single step of the in vitro fertilization process. Furthermore, experimentation are seldom conducted on human oocytes. Developments including more than one accurate system performing the different crucial tasks needed are rare. This issue is addressed in this paper by the development of smart microrobots. The first device performs mechanical cell characterization for oocyte sorting while the second performs cell convoying for non-invasive cell transporting.

\section{MechanicAl CELl CHARACTERIZATION DEVICE}

The micro-force sensor (figure 1) consists of a cylindrical glass tip $(120 \mathrm{~mm}$ long and $500 \mu \mathrm{m})$ which levitates in a magnetic field produced by four $\mathrm{NdFeB}$ magnets (called $M_{1}$ ). Two cylindrical NdFeB magnets (ForceField), called $M_{2}$, are fitted to the glass tip. The force sensor configuration can be considered as two frictionless magnetic bearings called $L 1$ and $L 2$ respectively (cf. figure 1 ). To ensure stable levitation and overcome unstable forces, the cylindrical magnets as well as 


\begin{tabular}{|c|c|c|c|}
\hline & Material & $\begin{array}{l}\text { Magnetic } \\
\text { property }\end{array}$ & Dimension \\
\hline Magnet M1 & $\mathrm{NdFeB}$ & $B_{r}=1.3 \mathrm{~T}$ & $\begin{array}{c}10 \mathrm{~mm} \mathrm{x} \\
10 \mathrm{~mm} \mathrm{x} \\
10 \mathrm{~mm}\end{array}$ \\
\hline Magnet M2 & $\mathrm{NdFeB}$ & $B_{r}=0.95 \mathrm{~T}$ & $\begin{array}{c}\phi 1.63 \\
\mathrm{~mm} \mathrm{x} \\
2.34 \mathrm{~mm}\end{array}$ \\
\hline $\begin{array}{l}\text { levitating } \\
\text { part }\end{array}$ & Glass & - & $\begin{array}{l}\phi 0.5 \mathrm{~mm} \\
\times 95 \mathrm{~mm}\end{array}$ \\
\hline $\begin{array}{l}\text { Tip of the } \\
\text { endeffector }\end{array}$ & Glass & - & $\begin{array}{c}5 \mathrm{~mm} \times \phi \\
0.02 \mathrm{~mm}\end{array}$ \\
\hline $\begin{array}{c}\text { Diamagnetic } \\
\text { material }\end{array}$ & Graphite & $\chi_{m}=-12 \mathrm{e}^{-5}$ & $\begin{array}{c}40 \mathrm{~mm} \mathrm{x} \\
40 \mathrm{~mm} \mathrm{x} \\
10 \mathrm{~mm}\end{array}$ \\
\hline
\end{tabular}

TABLE I

COMPONENTS OF THE FORCE SENSING DEVICE.

the tip are placed between two plates of a diamagnetic material (graphite from ForceField). According to the magnetic polarity arrangement (cf. figure 2), magnets $M_{1}$ induce attractive forces on magnets $M_{2}$. Without using a diamagnetic material the latter configuration of levitation is unstable. In fact, the magnetic properties of graphite ensure stable levitation of magnets $M_{2}$ and thus the stability of the cylindrical glass tip position. A laser sensor (ELITEC Telemeter) is used to monitor the displacement of the sensing part along the longest length of the cylindrical glass tip. Table I shows the main characteristics of the force sensing device components.

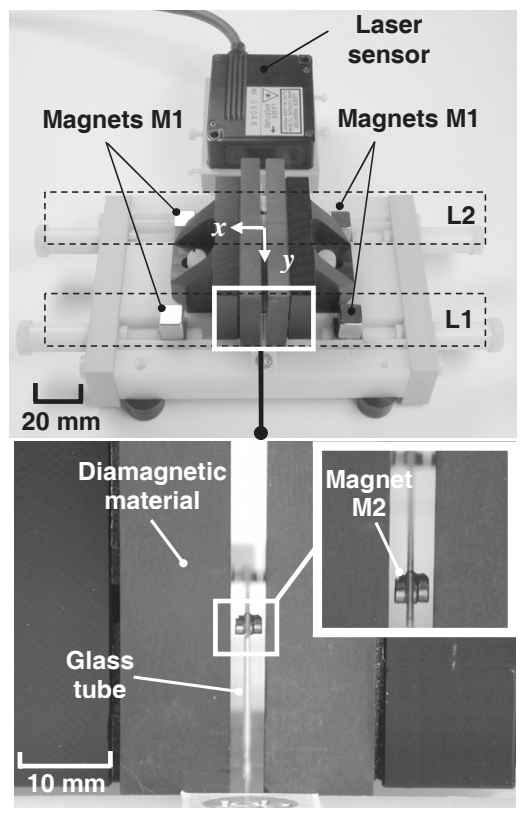

Fig. 1. The frictionless force sensor overview. The frictionless magnetic bearing $L 1$ and $L 2$ ensure a passive stable levitation of the cylindrical glass tip. A telemeter laser sensor is used to monitor the displacement of the sensing part.

\section{A. Force measurements model}

The attractive magnetic forces $\vec{F}_{u}^{m}$ (cf. figure 2) along a direction $\vec{u}(\vec{u} \in\{\vec{x}, \vec{y}, \vec{z}\})$ can be expressed in cartesian coordinates according to both remanent magnetic induction $B_{r}$ of the magnet $M_{2}$ and the magnetic induction $B_{1}$ produced by the magnets $M_{1}$ as [12]

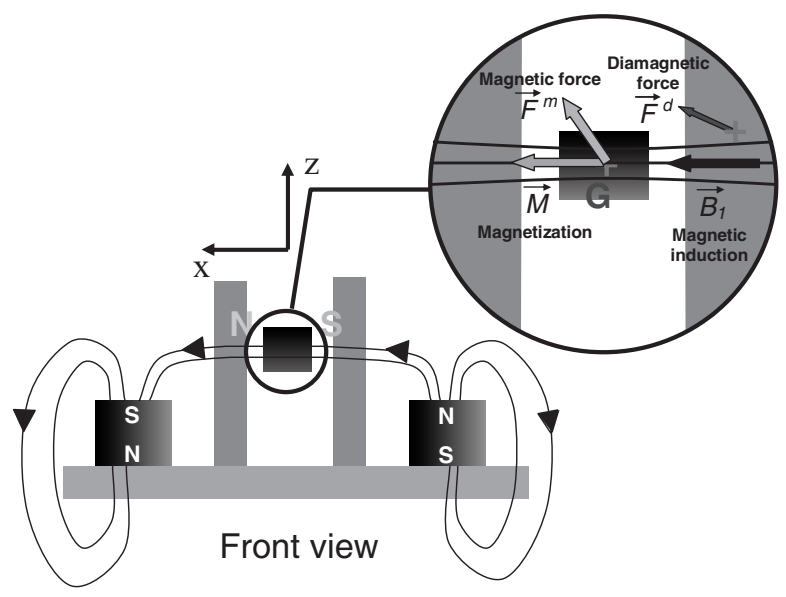

Fig. 2. Magnetic polarity arrangement and forces scheme interaction. The magnets $M_{1}$ induce attractive forces on the magnet $M_{2}$. The stable levitation configuration is ensured by means of the graphite (diamagnetic material).

$$
\vec{F}_{u}^{m}=\frac{V B_{r}}{2 \mu_{0}\left\|\vec{B}_{1}(G)\right\|} \vec{\nabla}_{u}\left\|\vec{B}_{1}(G)\right\|^{2}
$$

Where $B_{1}(G)$ is the magnetic induction produced by $M_{1}$ at the center of gravity $G$ of $M_{2}, \vec{\nabla}$ is the nabla operator $\left(\vec{\nabla}=\left(\frac{\partial}{\partial x}, \frac{\partial}{\partial y}, \frac{\partial}{\partial z}\right)^{T}\right), \mu_{0}$ the vacuum permeability and $V$ the volume of $\mathrm{M}_{2}$.

The repulsive diamagnetic forces $\vec{F}_{u}^{d}$ (cf. figure 2) along a direction $\vec{u}$ produced by a small element $P$ of the graphite on $M_{2}$ can be expressed in cartesian coordinates according to both dimensionless scalar magnetic susceptibility $\chi_{m}$ and the magnetic induction $B_{2}(P)$ produced by the magnets $M_{2}$ as [12]

$$
\vec{F}_{u}^{d}=\frac{\chi_{m}}{2 \mu_{0}} \iiint_{v^{\prime}} \vec{\nabla}_{u}\left\|\vec{B}_{2}(p)\right\|^{2} d v^{\prime}
$$

\section{B. Magnetic model validation}

Since the magnetic and diamagnetic forces are expressed as a function of the magnetic induction produced by the magnets configuration $M_{1}$ as well as the magnets $M_{2}$, the determination of $\vec{B}_{1}$ and $\vec{B}_{2}$ are crucial. Hence, the more accurate the determination of the $\vec{B}_{1}$ and $\vec{B}_{2}$ the more accurate is the force measurements process. For this reason, we used a finite element analysis method achieved with Flux3D software. Figure 3 shows the simulated magnetic induction produced by a single magnet $M_{1}$ at different heights $z$ and compared to the averaged experimental measurements achieved by means of a calibrated Hall sensor (F.W. BELL Teslameter). The experimental measurements are performed by means of a servomotor 
which accurately move the probe above the magnet. In the present validation study, the travel range and the resolution step measurements are $20 \mathrm{~mm}$ and $1 \mu \mathrm{m}$ respectively. We observed a small deviation between the simulated and the experimental curves (a relative error of less than 1\%). The results show good agreement between the simulated and the experimental methods and demonstrate, in our case, the efficiency of the finite element analysis method.

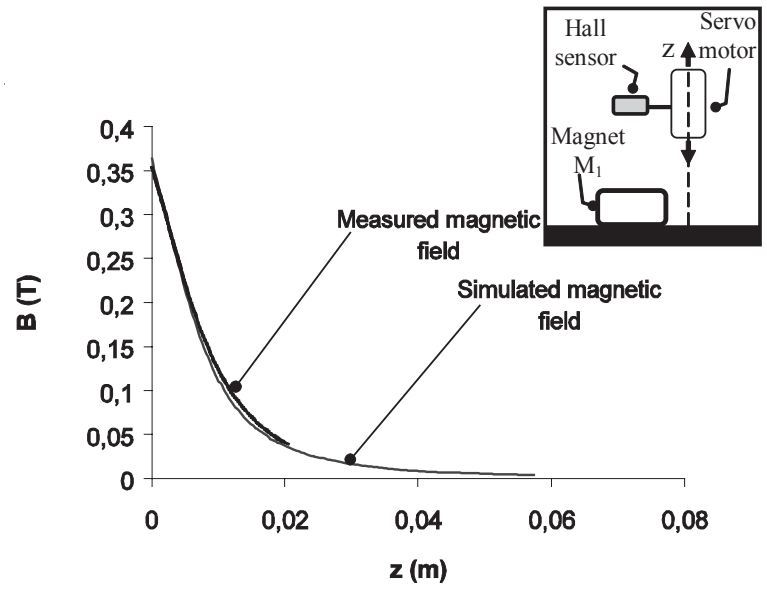

Fig. 3. Simulated magnetic induction produced by a single magnet $M_{1}$ compared to averaged experimental measurements.

\section{Force calibration}

The calibration of the developed force sensor is performed by means of a compression calibrated load cell (Adventure AR0640, OHAUS). The latter is able to detect weight variations of less than $50 \mu \mathrm{g}$. Since the detection range of the load cell is bigger than the developed device, we have performed force calibration in the range of $1 \mu N$ to $20 \mu N$. For force calibration out of detection range, we have used the magnetic model. In fact, equation 1, gives an accurate and quantitative information about the force magnitude based on the accurate determination (using the calibrated Hall sensor F.W. BELL Teslameter) of the magnetic induction $\vec{B}_{1}$ produced by the magnets $M_{1}$.

\section{OOCYTE CONVEYOR SYSTEM}

The aim of the oocyte conveyor system is to perform cell transport (see figure 4). Our original cell transport system consists of a wireless soft ferromagnetic micropusher which pushes a single cell. This soft ferromagnetic particle $(200 \times$ $200 \times 20 \mu \mathrm{m}^{3}$ ) is made of electroplated nickel as described in [13] and is actuated by an external magnetic field. This magnetic field is induced by a cylindrical magnet $(\mathrm{NdFeB}, \phi$ $1.6 \mathrm{~mm}, \mathrm{~h}=0.74 \mathrm{~mm}$ ) placed in the external medium on the other side of a vertical wall (see in figure 4). The magnet displacements are performed by two motorized translation stages (M-111.2, Physik Instrumente) controlled by computer. The micropusher placed against the wall is used to place oocytes in front of tools such as an injector, aspiration pipe or force sensor described in section I, etc. Contrary to fluid flow transfer, the micropusher can push one cell without modifying the position of other cells under treatment. The first Degree Of Freedom DOF (lateral movement in figure 4) is used to push the cells. The second DOF (vertical movement in figure 4 ) is used to go over the cells. At the present time the device is not able to change the pushers.
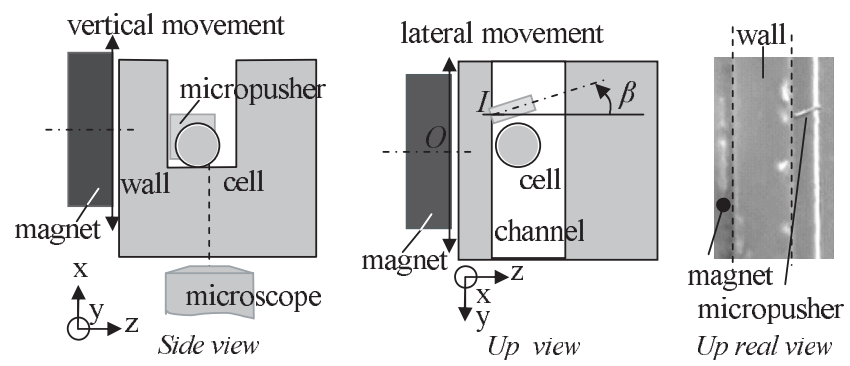

Fig. 4. Magnetic Cell Micropusher Principle: Cells are pushed in a channel by a ferromagnetic micropusher which follows the movement of a permanent magnet. Permanent magnet is moved in two directions ( $x$ : vertical movement and $y$ : lateral movement). Consequently, the pusher is able to push a cell along $y$ axis and to go over a cell along $x$ axis.

The micropusher behavior is divided into two types: rotation around the contact point and translation of the contact point. As the angular behavior is a keypoint which allows precise positioning [14], this paper focuses on the angular model.

The magnet position is characterized by the reference $(O, \vec{x}, \vec{y}, \vec{z})$ defined in figure 4 . The pusher position is defined by the point $I$, center of the contact line between the pusher and the wall. Micropusher orientation is defined by the angles $\alpha$ and $\beta$ respectively around $\vec{z}$ and $\vec{x}$.

\section{A. Angular Behavior Modeling}

Angular behavior is given by the magnetic efforts applied by the magnet on the micropusher. The magnetic torque $\overrightarrow{d \Gamma_{m}}(P)$ and magnetic force $\overrightarrow{d F_{m}}(P)$ applied on an elementary volume $d v$ in point $P$ is considered as

$$
\left\{\begin{array}{c}
\overrightarrow{d \Gamma_{m}}(P)=\vec{M} \wedge \overrightarrow{B_{0}} \cdot d v \\
\overrightarrow{d F_{m}}(P)=\nabla\left(\vec{M} \times \overrightarrow{B_{0}}\right) \cdot d v
\end{array}\right.
$$

where $\overrightarrow{B_{0}}$ is the magnetic field of the permanent magnet calculated by the Finite Element Model FLUX3D and $\vec{M}$ is the internal magnetization which represents the magnetic behavior of the soft ferromagnetic micro-pusher. To determine the magnetization, two hypotheses are assumed: The micropusher is a flat surface $S$; the magnetization module reached the magnetic saturation $M_{\text {sat }}=5.1 \times 10^{5} \mathrm{~A} \cdot \mathrm{m}^{-1}$. Magnetization orientation can be calculated classically by computing the well-known continuity relation of the magnetic field on the surface $S$ as described in [15].

Considering the very low inertia of micro-objects, the angular dynamic time constant of the micro-pusher is in the order of $10 \mu \mathrm{s}$, thus we consider only the static position given by

$$
\iint_{V} \overrightarrow{d \Gamma_{m}}(P)=-\iint_{V} d \overrightarrow{F_{m}}(P) \wedge \overrightarrow{P I}
$$




\section{B. Experimental Validation}

Orientation is determined by the equality between both terms of (4). These two strains induce two different behaviors. The magnetic torque $\overrightarrow{\Gamma_{m}}$ consequence is the alignment of the micropusher on the external magnetic field $\overrightarrow{B_{0}}$. In contrast, the magnetic force torque $\overrightarrow{F_{m}} \wedge \overrightarrow{P I}$ makes the micropusher lie flat on the wall. Both phenomena have the same value order and micropusher orientation is defined by the equilibrium of both physical effects.

The experimental measurements and simulations results are presented in figure 5. The experimental conditions are $\overrightarrow{O I} \cdot \vec{x} \in[-500 ; 500] \mu m, \overrightarrow{O I} \cdot \vec{y}=0, \overrightarrow{O I} \cdot \vec{z}=200 \mu m$. As presented in figure 5, experimental measurements and the model are similar.

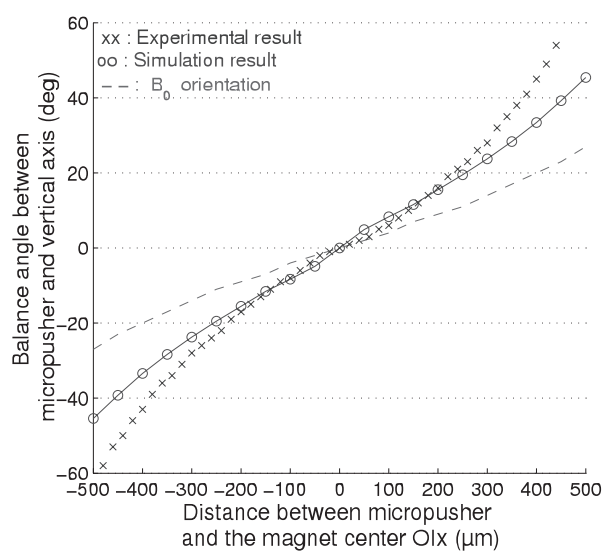

Fig. 5. Experimental and Simulated Micropusher Angles $\beta$ : Orientation $\beta$ is presented in function of the relative position between the micropusher and the magnet $O I_{x}$. Experimental and simulated orientations are relatively near. The micropusher orientation is different from the orientation of the magnet field $B_{o}$ around the micropusher: The micropusher is not aligned on magnetic field lines.

The alignment of the ferromagnetic objects on the magnetic field is a specificity of the microworld. In fact, the scale effect on both phenomena is different: magnetic torque $\overrightarrow{\Gamma_{m}}$ is a function of $l^{3}$ while magnetic force torque $\overrightarrow{F_{m}} \wedge \overrightarrow{P I}$ is a function of $l^{4}$ (with $l$ the micropusher characteristic size). Consequently, the smaller the micropusher the closer its orientation is to the magnetic field line.

\section{BIOLOGICAL EXPERIMENTATIONS}

\section{A. Cell culture}

The oocytes are prepared on Petri dishes with specific culture medium formed by Dulbecco's Modified Eagle's Medium (DMEM) with high glucose and L-glutamine components and $10 \%$ of foetal bovine serum. The oocytes can be assimilated morphologically to an spherical cells with a thin surrounding biomembrane (100 - $150 \mu \mathrm{m}$ radius). The cell observation is performed by means of an inverted microscope (Nikon) with 60x magnification lens.

In the present study, the experiments are conducted at ambient conditions on non-fertilized human oocytes which have not been selected for ICSI process. The oocytes are from the research group on genetics and reproduction at the Besançon Hospital Center (France).

\section{B. Oocyte mechanical characterization experiments}

Figure 6 shows the overview of the developed non-invasive process for mechanical cell characterization. Instead of moving the force sensing device, we choose to keep it immobile. Accordingly, oocytes assigned for mechanical characterization study are arranged on Petri dishes with a glass head. The latter are positioned above the micropositioning stage. No holding pipette is used for the mechanical characterization process in order to reduce tensile strain. Hence, the oocytes are squeezed against a glass slide. Figure 6 shows the mechanical characterization process.

Preliminary mechanical experiments are conducted on human oocytes not selected for in vitro fertilization process. First mechanical characterization experimentation is focused on the estimation of the stiffness $K$ of the biological sample as well as on Young's Modulus E. Since Young's modulus can be used to predict the elongation or compression of elastic samples as long as the stress is less than the yield strength of the sample, mechanical characterization experiments on the biological sample are restricted to mechanical behavior where elastic linear properties are satisfied. Figure 7(A) shows experimental measurements of the oocyte deformation $\delta$ as a function of the applied force $F$. According to figure 7(A) the linear elastic behavior is satisfied for forces less then $0.2 \mu N$. Based on these assumptions, the stiffness $K$ of the biological sample can be expressed by a linear analytical formula $(F=$ $K \delta$ ). Hence, the stiffness of the biological sample is found in order of $K=0.015 \mathrm{~N} / \mathrm{m}$. Young's Modulus $E$ is estimated using Hook's law $(\sigma=E \varepsilon)$. Figure 7(B) shows the stress $\sigma=\frac{F}{a}$ as a function of the dimensionless strain $\varepsilon=\frac{\delta}{2 R}$. Using linear interpolation function, Young's Modulus is found equal to $E=0.14 M P a$.

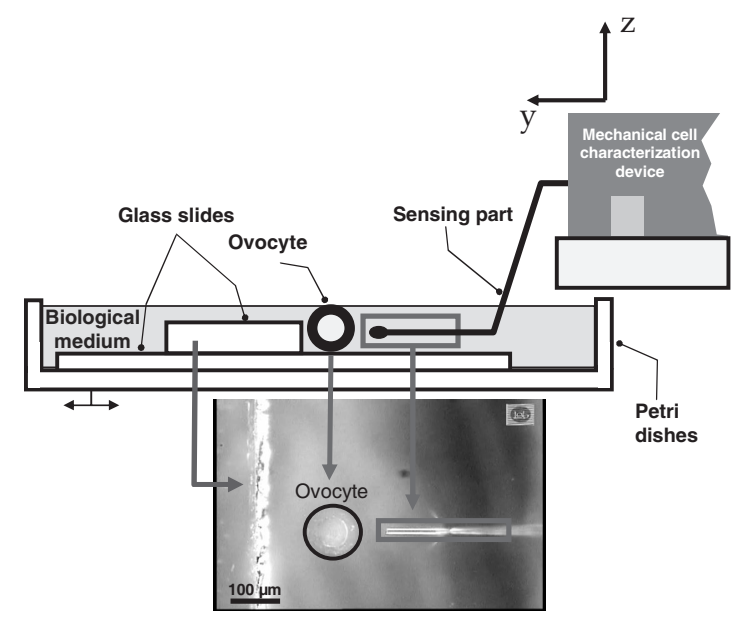

Fig. 6. Overview of the developed non-invasive process which squeeze the oocyte against a glass slide. No holding pipette is used for the mechanical characterization process in order to reduce tensile strain. The displacement of the sensing part is monitored by means of the laser sensor.

\section{Oocyte pushing operation}

Some open loop pushing operations were undertaken with the micropusher without force feedback. However, the force 

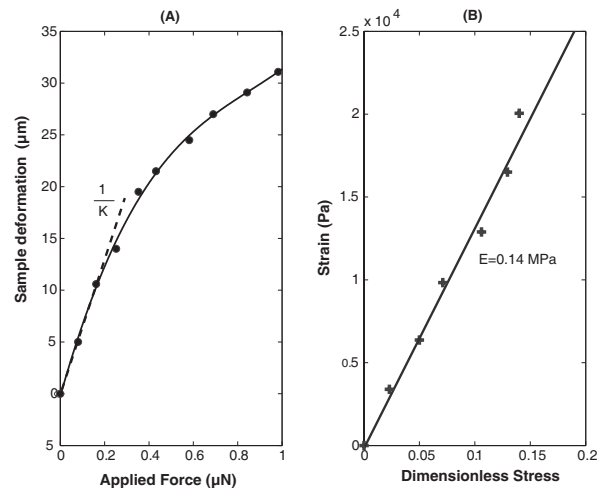

Fig. 7. (A) Sample deformation $\delta$ as a function of the applied force $F$ (B) Stress $\sigma=E \varepsilon$ as a function of the dimensionless strain $\varepsilon=\frac{\delta}{2 R}$.

applied by the pusher on the cell can be estimated by the measurement of the relative position between the micropusher and the magnet as described in [16].

An example of human oocyte micromanipulation is presented in figure 8. The Oocyte is pushed from one workstation to another through the channel (horizontal motion in figure 8). The maximal velocity is $100 \mu m . s^{-1}$. During the pushing operations, we never observe sticking effects between the micropusher and the oocyte. At the present time the micropusher is made of nickel material, which is not biocompatible. However, these experiments on biological cells validate our concept of a new magnetic cell transport system.
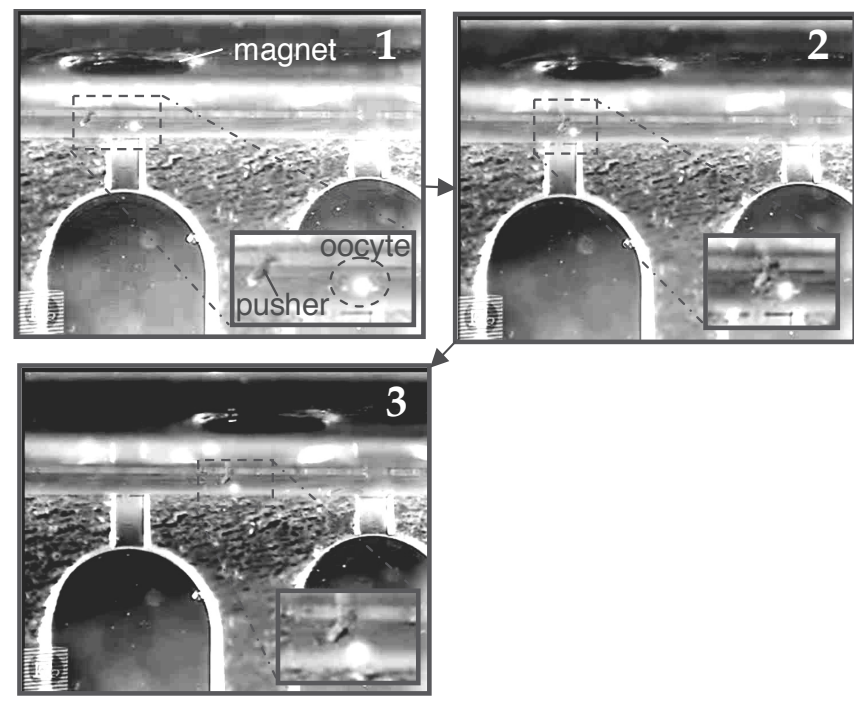

Fig. 8. Pushing Operation of a Human Oocyte: Experimental validation of the micropusher is performed on human oocytes. A cell is placed in the channel and manipulated by teleoperation. The lateral movement of the permanent magnet induces the displacement of the micro-pusher in the channel. Thus, the cell is transfered in the channel.

\section{CONCLUSION}

The paper has presented the description of two microrobot devices based on passive magnetic foundations: the mechanical cell characterization device based on forces sensing and the oocyte conveyance device based on non-invasive transporting approach. The design, calibration and the mechanical behavior of these devices are detailed. The results show good agreement between the simulated and the experimental data. The efficiency of the microrobotics systems is proved by successful in vitro experiments on human oocytes. Hence, accurate mechanical characterization for oocyte sorting criterion investigation is achieved as well as non-invasive transporting and positioning tasks.

\section{ACKNOWLEDGMENTS}

This research was supported by the LAB (Laboratory of Automation of Besançon - France). The authors thank Prof. C. Roux (research group on "genetics and reproduction", Besançon) for collaboration on human oocyte micromanipulations. The authors thank the LCEP-Besançon for the fabrication of the micropusher glass sample support.

\section{REFERENCES}

[1] Deok Ho Kim, Chang Nam Hwang, Yu Sun, Sang Ho Lee, Byungkyu Kim, and Nelson B.J. Mechanical analysis of chorion softening in prehatching stages of zebrafish embryos. IEEE Transactions on NanoBioscience, 5(2):89 - 94, June 2006.

[2] Yu Sun, Wan K.T., Roberts K.P., Bischof J.C., and Nelson B.J. Mechanical property characterization of mouse zona pellucida. IEEE Transactions on NanoBioscience, 2(4):279 - 286, Dec. 2006.

[3] Xudong Li, Guanghua Zong, Shusheng Bi, and Wei Zhao. Automatic micromanipulating system for biological applications with visual servo control. Journal of Micromechatronics, 1(4):345-63, 2002.

[4] X. Xiong, Yael Hanein, J. Fang, D. T. Schwartz, and K. Böhringer Multi-batch self-assembly for microsystem integration. In Proc. Of the 3rd Int. Workshop on Microfactories - IWMF02, pages 25-28, Minneapolis - USA, sept. 2002.

[5] M. Frenea, S. P. Faure, B. Le Pioufle, Ph. Coquet, and H. Fujita. Positioning living cells on a high-density electrode array by negative dielectrophoresis. Materials Science and Engineering, 23:597-603, 2003.

[6] B. K. Kim, D. H. Kim, J. Park, Y. Kim, S. J. Kwon, H. Kang, and S. H. Jung. Autonomous biomanipulation factory for manipulating individual embryo cells. In Proceedings of the Workshop Microrobotics for biomanipulation, IROSO3, pages 74-88, Las Vegas, USA, Oct 2003.

[7] Y. Sun, M. A. Greminger, and B. J. Nelson. Investigating protein structure with a microrobotic system. In Proceedings of the 2004 IEEE ICRA, pages 2854-59, New Orleans, USA, April 2004.

[8] A. Ichikawa, F. Arai, H. Maruyama, T. Fukuda, and T. Katsuragi. Single cell trap on a chip using in-situ microfabrication with photocrosslinkable resin and thermal gelation. In Proceedings of the 2004 IEEE ICRA, pages 2848-53, New Orleans, USA, April 2004.

[9] D-H Kim, B. Kim, S. Yun, and S. Know. Cellular force measurement for force reflected biomanipulation. In Proceedings of the 2004 IEEE ICRA, pages 2412-17, New Orleans, USA, April 2004.

[10] F. Arai, K. Motoo, T. Fukuda, and T. Katsuragi. High sensitive micro touch sensor with piezoelectric thin film for micropipetting works under microscope. In Proceedings of the 2004 IEEE ICRA, pages 1352-57, New Orleans, USA, April 2004.

[11] M. Gauthier and E. Piat. An electromagnetic micromanipulation system for single cell manipulation. Journal of Micromechatronics, 2(2):87119, Feb 2004

[12] M. Boukallel, E. Piat, and J. Abadie. Passive diamagnetic levitation: theoretical foundations and application to the design of a micro-nano force sensor. In Proceedings of the IEEE International Conference on Intelligent Robots and systems, pages 1062-1067, Las Vegas, USA, oct. 2003.

[13] M. Gauthier and E. Piat. Microfabrication and scale effect studies for a magnetic micromanipulation system. In Proc. of the IEEE International Conference on Intelligent Robots and Systems - IROSO2, volume 2, pages 1754-59, Lausanne - Switzerland, 30 sept - 4 Oct 2002.

[14] M. Gauthier and E. Piat. Control of a particular micro-macro positionning system applied to cell microdisplacement. IEEE Transactions on Automation Science and Engineering, 3(3):264-271, 2006. 
[15] M. Dauge, M. Gauthier, and E. Piat. Modelling of a $2 d$ magnetic cell transport system. In proc. of the IEEE Int. Conf. on Intelligent Robots and Systems, pages 4098-4103, August 2005.

[16] M. Gauthier and E. Piat. Force study applied by a planar micromanipulator to biological objects. Journal Européen des Systèmes Automatisés, 36(9):1249-64, december 2002. 